\title{
Prevalência e tipo de aleitamento materno entre mulheres com alto risco gestacional
}

\author{
Prevalence and breastfeeding type among high-risk pregnancy women \\ Prevalencia y tipo de lactancia materna entre mujeres con alto riesgo gestacional \\ Aline Silva de Andrade ${ }^{1}$, Karla Oliveira Marcacine ${ }^{1}$, Ana Cristina Freitas de Vilhena Abrão ${ }^{1}$, Kelly Pereira Coca $^{1,2}$
}

\section{Resumo}

Objetivo: Identificar a prevalência e o tipo de aleitamento praticado no primeiro e quarto mês após o parto por mulheres que apresentaram gestação de risco.

Métodos: Coorte prospectiva com dados secundários realizado em um ambulatório de aleitamento materno, São Paulo. A amostra foi composta por prontuários de mulheres e seus filhos atendidos entre 2004 e 2016. A presença de doença materna foi considerada independente do momento do diagnóstico, antes ou durante a gestação, e 0 tipo de AM praticado no $1^{\circ}$ e $4^{\circ}$ mês pós-parto.

Resultados: Analisou-se 1.607 prontuários, $27 \%$ das mulheres estudadas apresentaram algum tipo de doença na gestação. A prevalência de AME foi cerca de $78 \%$ no $1^{\circ}$ e de $63 \%$ no $4^{\circ}$ mês pós-parto, e o tipo de AM praticado foi semelhante entre e os grupos no $1^{\circ}(p=0,69)$ e $4^{\circ}$ mês $(p=0,19)$ pós-parto.

Conclusão: A presença de doença na gestação não interferiu na taxa de aleitamento materno exclusivo praticado na população estudada.

\section{Abstract}

Aim: To identify the prevalence and the type of breastfeeding practiced in the first and fourth month after delivery by women who presented a high-risk pregnancy.

Methods: Prospective cohort with secondary data performed in an outpatient breastfeeding, São Paulo. The sample was composed of medical records of women and their children attended between 2004 and 2016. The presence of maternal illness was considered independent from the time of diagnosis, before or during pregnancy, and the type of $\mathrm{BF}$ practiced was identified at the $1^{\text {st }}$ and $4^{\text {th }}$ month postpartum.

Results: 1,607 examined medical records, $27 \%$ of women had some kind of disease while they were pregnant. The prevalence of the exclusive breastfeeding was about $78 \%$ in the first and $63 \%$ in the fourth month postpartum, and the type of breastfeeding practice was similar in both groups at the $1^{\text {st }}(p=0.69)$ and $4^{\text {th }}$ month $(p=0.19)$ postpartum.

Conclusion: The presence of illness in pregnancy did not change exclusive breastfeeding rate in the population studied.

\section{Resumen}

Objetivos: Identificar la prevalencia y el tipo de lactancia practicado en el primer y cuarto mes después del parto por mujeres que presentaron gestación de alto riesgo.

Métodos: Cohorte prospectiva con datos secundarios realizado en un servicio de lactancia en São Paulo. La muestra fue obtenida por registros de las consultas de mujeres y sus hijos asistidos entre 2004 y 2016. La presencia de enfermedad materna fue considerada independiente del momento da descubierta de la enfermedad, antes o durante el embarazo, y el tipo de la lactancia ocurrió en el $1^{\circ}$ y $4^{\circ}$ mes después del parto.

Resultados: 1.607 registros de consulta fueran analizados, $27 \%$ de las mujeres estudiadas presentaron algún tipo de enfermedad en el embarazo. La prevalencia de lactancia exclusiva fue de $78 \%$ en el primer mes y $63 \%$ en el cuarto mes después del parto, y el tipo de la lactancia practicada fue similar entre los grupos en el $1^{\circ}(p=0.69)$ y $4^{\circ}$ mes $(p=0,19)$ después del parto.

Conclusión: La presencia de enfermedad en el embarazo no cambió la tasa de lactancia materna exclusiva en la población estudiada.

\section{Descritores}

Aleitamento materno; Gravidez de alto risco; Diabetes Mellitus; Hipertensão; Desmame

\section{Keywords}

Breastfeeding; High-risk pregnancy;

Diabetes Mellitus; Hypertension; Weaning

\section{Descriptores}

Lactancia materna; Embarazo de alto riesgo; Diabetes Mellitus; Hipertensión; Destete

\section{Como citar:}

Andrade AS, Marcacine KO, Abrão AC, Coca KP. [Prevalence and breastfeeding type among high-risk pregnancy women]. Rev Soc Bras Enferm Ped. 2018;18(2):90-5. Portuguese

${ }^{1}$ Escola Paulista de Enfermagem, Universidade Federal de São Paulo, São Paulo, SP, Brasil.

2 School of Nursing, La Trobe University, Melbourne, Austrália.

Conflitos de interesse: nada a declarar.

Submissão: 9 de Novembro de 2018 | Aceite: 27 de Junho de 2019

Autor correspondente: Kelly Pereira Coca | Rua Napoleão de Barros, 754, Vila Clementino, São Paulo, SP, Brasil. kcoca@unifesp.br

https:// orcid.org/0000-0002-3604-852X

DOI: http://dx.doi.org/10.31508/1676-3793201800014 


\section{Introdução}

O aleitamento materno é recomendado de forma exclusiva (AME) de zero a seis meses e complementado por dois anos por ser considerado como a alimentação ideal para as crianças. ${ }^{(1)}$ Essa recomendação justifica-se pelos diversos benefícios que a prática oferece, tanto para a criança como para a mulher. ${ }^{(2)}$ Para a criança, proporciona proteção contra as doenças mais prevalentes do primeiro ano de vida, como a diarreia e as infecções respiratórias; e oferece benefícios a longo prazo ao reduzir o risco de doenças crônicas da idade adulta tais como: alergias, hipertensão, hipercolesterolemia, diabetes e obesidade. ${ }^{(2)}$ No que se refere à saúde da mulher, previne a ocorrência de hemorragia pós -parto, de doenças cardiovasculares e das síndromes metabólicas; reduz os riscos de canceres de mama e de ovário e favorece o retorno ao peso pré-gestacional. ${ }^{(2,3)}$

Apesar dos evidentes benefícios e do aumento da prevalência do aleitamento materno (AM) no Brasil nas últimas décadas, suas taxas ainda se mostram distantes ${ }^{(4,5)}$ das metas estabelecidas pela Organização Mundial de Saúde (OMS). ${ }^{(1)}$ Dentre os fatores relacionados ao desmame precoce, observam-se o baixo nível educacional materno, presença de dor/lesão mamilar, produção láctea insuficiente, cesariana, parto prematuro e as condições de saúde da mulher. ${ }^{(6,7)}$ No que diz respeito à saúde materna, sabe-se que a presença de doenças materna pode contribuir para um desfecho perinatal negativo, especialmente quando complicações associadas à internações previas ao parto acontecem. ${ }^{(8)}$ Estudiosos têm relatado que mulheres com doenças como sobrepeso ou obesas apresentam menores taxas de início e duração do AM ao longo do primeiro ano de vida da criança, e são menos propensas a amamentar exclusivamente, mesmo quando há o ajuste de variáveis interferentes nessa prática (idade, paridade, tipo de parto, tabagismo, intenção em amamentar e história pregressa de lactogênese tardia). ${ }^{(9)}$ Várias razões têm sido propostas para justificar a correlação entre o excesso de peso e a menor probabilidade de amamentar, entre elas, destacam-se o atraso no processo de lactogênese II, o desconforto com a própria imagem corporal e a menor autoconfiança materna em relação a capacidade de produzir leite suficiente. Além disso, algumas mulheres obesas têm mamas muito volumosas, o que pode dificultar o posicionamento da criança e, podem ainda, apresentar um aumento do edema pós-parto, o que aplaina os mamilos, tornando mais difícil a pega da mama pela criança. ${ }^{(10,11)}$

Assim como o excesso de peso, a Diabetes materna também parece interferir negativamente na lactação, devido a hiperinsulinemia fetal e, consequente aumento do risco de hipoglicemia neonatal, favorecendo a introdução precoce de fórmulas infantis. ${ }^{(12,13)}$ Além disso, estudo americano demonstrou que $12 \%$ a mais das mulheres com Diabetes Mellitus Gestacional (DMG) relataram redução da produção láctea quando comparadas àquelas que não apresentavam tal doença. ${ }^{(12)}$ As síndromes hipertensivas da gestação também têm sido associadas ao início tardio e à descontinuidade precoce da amamentação. ${ }^{(14)}$ Pesquisa de coorte prospectiva, publicada em 2018, envolvendo 168 duplas mãe-filho, sendo 124 compostos por mulheres normotensas e 42 com hipertensas, observou que aquelas com hipertensão apresentaram maiores dificuldades em manter o aleitamento materno exclusivo (AME) seis meses após o parto, quando comparadas às mães normotensas. ${ }^{(15)}$

Considerando a tendência global contínua de aumento das doenças crônicas tanto na população geral de mulheres quanto aquelas na fase reprodutiva, ${ }^{(16)}$ bem como, os benefícios do aleitamento materno para a mulher, criança e sociedade ${ }^{(1)}$ e as possíveis dificuldades vivenciadas pelas puérperas com doenças maternas ao amamentar, torna-se de particular importância o conhecimento das taxas de aleitamento praticado nesta população em específico, de modo a oferecer uma assistência adequada desde a gestação até o pós-parto, facilitando o início e manutenção da amamentação. ${ }^{(13)}$

Nesse contexto, o presente estudo buscou identificar a prevalência e o tipo de aleitamento praticado no primeiro e quarto mês após o parto por mulheres que apresentaram gestação de risco.

\section{Métodos}

Coorte prospectiva com dados secundários de um ambulatório de seguimento, recorte de uma pesquisa maior intitulada "A repercussão do atendimento em serviço especializado na prevalência de aleitamento materno". O estudo foi realizado em um ambulatório de aleitamento materno de São Paulo, Brasil, que ofe- 
rece atendimento diário para a promoção e acompanhamento do aleitamento materno de mulheres e seus filhos, por meio de demanda espontânea e/ou daquelas que tiveram parto no Hospital Universitário - HU da Universidade Federal de São Paulo (UNIFESP), hospital de uma instituição de ensino público da cidade de São Paulo.

A amostra do estudo foi composta por prontuários de mulheres e seus filhos atendidos no período de 2004 e 2016. Excluiu-se aqueles referentes às mulheres com gestação múltipla, nascimento prematuro e/ou internação do recém-nascido na unidade de Terapia Intensiva Neonatal, que descontinuaram o seguimento após a primeira consulta e com registros incompletos. Os dados foram coletados no período de agosto a dezembro de 2017.

As variáveis obtidas para este estudo foram: dados de caracterização da mulher (idade escolaridade, situação conjugal, número de gestações, paridade e tipo de parto); dados da criança (sexo, Apgar no 5o minuto de vida e peso ao nascer); variáveis relacionadas à condição de saúde materna (presença e tipo de doença) e o tipo de aleitamento materno praticado. A presença de doença(s) materna(s) foi considerada independente do momento do diagnóstico, podendo ter sido desenvolvida antes ou durante o período gestacional. O tipo de AM praticado foi analisado no $1^{\mathrm{o}} \mathrm{e}$ no $4^{\circ}$ mês pós-parto, período justificado pelo retorno da maioria das mulheres ao trabalho após o $4^{\circ}$ mês de vida da criança e pela queda significativa de acompanhamento no ambulatório.

Os dados foram submetidos à análise descritiva, obtendo-se média e desvio padrão para as variáveis contínuas, e frequência e percentual para as variáveis categóricas. Para comparar a curva de sobrevida por ocorrência de doença durante a gestação, foi utilizado Kaplan-Meier, e para ajuste das curvas aplicou-se o teste de Breslow. Para a sobrevida considerou-se o tempo, em dias, até o término do aleitamento materno exclusivo. Para comparar a evolução da amamentação com ocorrência da doença durante a gestação foi utilizado Generalized Estimating Equation (GEE). Foi utilizado um nível de significância de 5\% (p-valor $<0,05$ ).

A pesquisa respeitou as recomendações da Resolução n. 466/12 do Conselho Nacional de Saúde e foi aprovada pelo comitê de Ética em Pesquisa da Universidade Federal de São Paulo sob número 2.362.050.

\section{Resultados}

Compuseram a amostra do estudo 1.607 prontuários de mães e seus respectivos filhos. As mulheres apesentavam uma média de $29(\mathrm{DP}=7)$ anos de idade, a maioria estudou até o ensino médio, $75,5 \%$ viviam com o companheiro, eram multigestas (média 2 gestações, $\mathrm{DP}=1,5)$, multíparas (média de 1.9 partos, $\mathrm{DP}=1$ ) e tiveram parto cesariana $(51,8 \%)$. As crianças estavam igualmente distribuídas em menos e meninos, 99\% deles receberam nota entre 8 e 10 no $5^{\underline{0}}$ minuto de vida e pesavam $3.057(\mathrm{DP}=603,11)$ gramas ao nascer, em média. Quanto às condições de saúde materna, 27,2\% delas apresentaram algum tipo de doença quando estavam gestantes, sendo a doença hipertensiva a mais frequente (Tabela 1).

Tabela 1. Presença e tipo de doença identificada entre as mulheres analisadas

\begin{tabular}{lc}
\hline Variável & $\mathrm{n}(\%)$ \\
\hline Doença materna $(\mathrm{n}=1607)$ & \\
Sim & $436(27)$ \\
Não & $1171(73)$ \\
Tipo de doença $(\mathrm{n}=432)^{*}$ & \\
Hipertensão & $204(47)$ \\
Diabetes Mellitus & $183(42,1)$ \\
Doenças infecciosas** & $29(6,8)$ \\
Outras*** & $18(4,1)$ \\
\hline
\end{tabular}

*Perda de dados; **Doenças infecciosas: hepatite B e C, sífilis, toxoplasmose, rubéola e citomegalovírus; ${ }^{* * *}$ Outras: obesidade $(\mathrm{n}=7)$, síndrome anti-fosfolípede $(\mathrm{n}=1)$, oligoamnio/ polidramnio $(\mathrm{n}=10)$

A prevalência de AME das crianças cujas mães apresentaram doença na gestação foi $78,4 \%$ no $1^{\circ}$ mês e $63,4 \%$ no $4^{\circ}$ mês pós-parto (Tabela 2). A prevalência de AME e não exclusivo entre as mulheres com e sem doença materna, foram similares nos dois intervalos estudados, não sendo observada diferença estatisticamente significante do tipo de aleitamento praticado entre os grupos analisados no $1^{\circ}(\mathrm{p}=0,69)$ e $4^{\circ}$ mês $(\mathrm{p}=0,19)$ após o parto (Tabela 2$)$.

Tabela 2. Tipo de aleitamento praticado no $1^{\circ}$ e $4^{\circ}$ mês após 0 parto, entre mulheres com e sem doença materna

\begin{tabular}{|c|c|c|c|c|}
\hline \multirow{3}{*}{$\begin{array}{l}\text { Variável } \\
\text { Doença } \\
\text { materna }\end{array}$} & \multicolumn{4}{|c|}{ Tipo de Aleitamento Materno } \\
\hline & \multicolumn{2}{|c|}{$\begin{array}{c}1^{0} \text { mês* } n / n^{\#} \\
(\%)\end{array}$} & \multicolumn{2}{|c|}{$\begin{array}{c}4^{0} \text { mês** } n / n^{\#} \\
(\%)\end{array}$} \\
\hline & AME & Não-AME & AME & Não-AME \\
\hline Sim & $\begin{array}{c}342 / 436 \\
(78,4)\end{array}$ & $\begin{array}{c}94 / 436 \\
(21,6)\end{array}$ & $\begin{array}{c}147 / 232 \\
(63,4)\end{array}$ & $\begin{array}{c}85 / 232 \\
(36,6)\end{array}$ \\
\hline Não & $\begin{array}{c}940 / 1171 \\
(80,3)\end{array}$ & $\begin{array}{c}231 / / 1171 \\
(19,7)\end{array}$ & $\begin{array}{c}378 / 590 \\
(64,1)\end{array}$ & $\begin{array}{c}212 / 590 \\
(35,9)\end{array}$ \\
\hline
\end{tabular}

AME - aleitamento materno exclusivo; * Média de 20 dias pós-parto $\left(\mathrm{n}^{*}=1.607\right) ; * *$ Média de 144 dias pós-parto $\left(\mathrm{n}^{\#}=822\right)$ 
A figura 1 apresenta o ajuste das curvas de sobrevida do grupo de mulheres com e sem doença materna, no qual observamos que não houve diferença significativamente na duração do AME praticado entre os grupos $(\mathrm{p}=0,88)$. As mulheres, independente do grupo, na sua maioria, deixaram de amamentar exclusivamente, em média, com 150 dias após o parto.

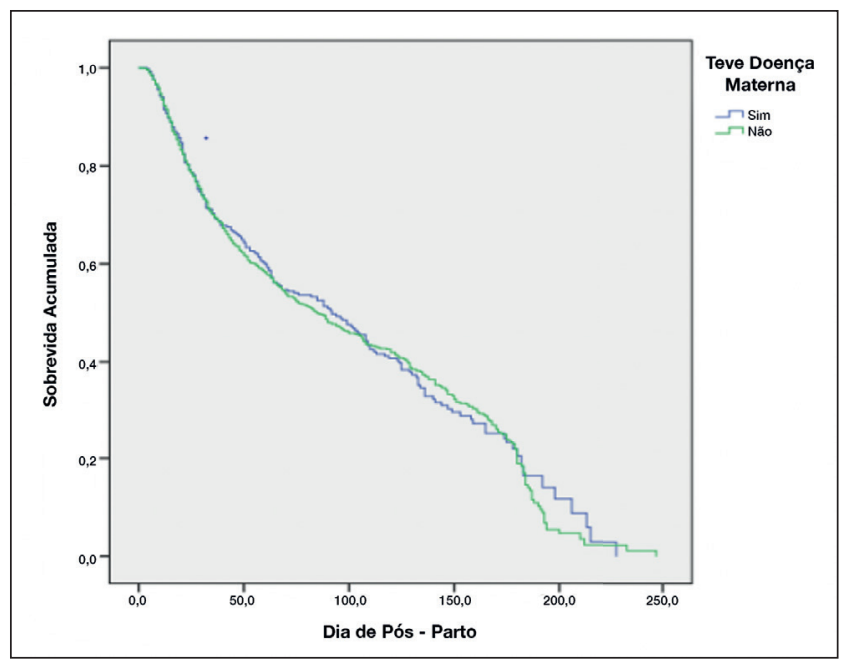

Figura 1. Curva de sobrevida ajustada por Kaplan-Meier por ocorrência de doença materna

\section{Discussão}

Este estudo identificou uma prevalência de 78\% e 63\% de AME entre mulheres que apresentaram doença na gestação, no $1^{\circ}$ e $4^{\circ}$ mês pós-parto respectivamente. Verificou-se que a presença de doença na gestação não interferiu no tipo e tempo de AME no período e população estudada.

Essas estimativas das prevalências são superiores àquelas descritas na última pesquisa realizada no Brasil, a qual demonstrou que, mais da metade das crianças, $60,7 \%$, é amamentada exclusivamente no primeiro mês de vida e essa proporção decresce para $23,3 \%$ aos 4 meses. ${ }^{(4)}$ A diferença encontrada pode justificar-se pela assistência do local do estudo, que oferece apoio e seguimento por profissionais qualificados, desde o nascimento até o sexto mês após o parto. No atual cenário das prevalências quanto à amamentação, o aconselhamento dos profissionais de saúde tem impacto significativo no auxílio à superação das dificuldades pré-estabelecidas. (17) $\mathrm{O}$ apoio deve ocorrer em diferentes momentos: no pré-natal, na sala de parto, no alojamento conjunto e no período puerperal pós alta hospitalar. ${ }^{(17)}$
No que se refere à prática do AME em mulheres com doenças, foram identificados apenas alguns estudos que investigaram o impacto de algumas doenças (síndromes hipertensivas, obesidade e DM), de forma isolada, e sua repercussão na gestação e na prática do aleitamento materno. ${ }^{(10-12,15,18)}$ Os resultados encontrados no presente estudo demonstram que somente a presença de doença materna não determina a duração do AME, pois trava-se de um processo complexo influenciado por aspectos não somente biológicos, mas também aqueles intrínsecos à mulher, relacionados ao seu estado afetivo, emocional e psíquico, além de fatores históricos, culturais, sociais e econômicos. ${ }^{(19)}$

Na população estudada, pode-se observar, em ambos os grupos, as características que estão relacionadas à proteção do AME, tais como: a idade materna, multiparidade e práticas de incentivo ao AM durante a internação hospitalar como a unidade de alojamento conjunto (AC), as quais podem ter contribuído para as taxas similares encontradas de AME. ${ }^{(20,21)}$ No que diz respeito à idade materna, apesar de não ser consensual na literatura, a faixa etária intermediária, de 20 a 34 anos, é apontada como a mais fortemente associada positivamente à prática e à duração do AME, ${ }^{(22,23)}$ assim como a multiparidade. ${ }^{(22,24)}$

É de senso comum que a prática adequada da amamentação exige conhecimento e preparo, e que a experiência prévia do AM pode propiciar a amamentação futura. ${ }^{(25)}$ Apesar de parte das mulheres analisadas apresentarem alguma doença materna, todas elas tiveram filhos em boas condições de nascimento e permaneceram em AC. Essa prática é considerada uma medida facilitadora para o início do AM, prevista pela Portaria MS/GM no 1.016/2003, ${ }^{(26)}$ e embasa-se nas vantagens de estimular o AM sob livre demanda, favorecer os laços afetivos entre mãe e filho por meio do convívio precoce e contínuo, prevenir infecção hospitalar e possibilitar o cuidado da criança pela sua mãe, para facilitar o reconhecimento das necessidades do filho e o esclarecimento de dúvidas com os profissionais de saúde, fatores essenciais para o êxito da amamentação.(21)

Nesse sentido, a disponibilidade de profissionais treinados para promoção e apoio ao AM durante a internação é fator fundamental para essa prática, especialmente em situações de dificuldades e/ou especiais, ${ }^{(27)}$ como na presença de doenças maternas. No que se refere às doenças infectocontagiosas, o conhecimento sobre as repercussões dessas doenças para a 
saúde da criança direciona para uma decisão esclarecida, com base teórica, em que a discussão acerca dos potenciais riscos versus os benefícios do aleitamento materno deve sempre ser levada em consideração.(28) Assim como as doenças infectocontagiosas, outras doenças como a hipertensão, o diabetes e a obesidade podem interferir no início e manutenção do AM, tanto pela separação do binômio devido à necessidade de internação da puérpera e/ou criança em Unidade de Terapia Intensiva, quanto pelo uso materno de medicações não compatíveis com essa prática. ${ }^{(9,12-14,29)}$

Apesar disso, as mulheres estudadas não apresentaram interferência da gestação de risco na prática do AM. Dessa forma, o suporte profissional adequado nessas situações é fundamental para prevenir o desmame ou a introdução desnecessária de fórmulas infantis. As limitações do estudo estão relacionadas à coleta de dados retrospectivos, além de ter sido desenvolvido em um único serviço de saúde com particularidades locais.

\section{Conclusão}

A presença de doenças na gestação não interferiu na taxa de aleitamento materno exclusivo praticado no $1^{\underline{0}}$ e $4^{\circ}$ mês após o parto. Conhecer a prevalência de aleitamento materno exclusivo entre puérperas com doenças pregressas e/ou desenvolvidas na gravidez e sua influência na duração do aleitamento materno exclusivo auxilia na compreensão da multicausalidade associada ao desmame precoce, bem como, reafirma a necessidade da implementação de estratégias que proporcionem a prática da amamentação.

\section{Agradecimentos}

De Andrade AS recebeu bolsa de pesquisa do Conselho Nacional de Desenvolvimento Científico e Tecnológico - CNPq por meio do Programa Institucional de Bolsas de Iniciação Científica da UNIFESP (2017-2018).

\section{Colaborações}

Andrade AS, Marcacine KO, Abrão ACFV e Coca KP contribuíram com a concepção do estudo, análise e in- terpretação dos dados, redação do artigo, revisão crítica relevante do conteúdo intelectual e aprovação da versão final a ser publicada.

\section{Referências}

1. World Health Organization. Global strategy for infant and young child feeding. Geneva: WHO; 2003.

2. Victora CG, Bahl R, BarrosAJ, França GV, Horton S, Krasevec J, et al. Breastfeeding in the 21st century: epidemiology, mechanisms, and lifelong effect. Lancet. 2016;387(10017):475-90.

3. Chowdhury R, Sinha B, Sankar MJ, Taneja S, Bhandari N, Rollins N, et al. Breastfeeding and maternal health outcomes: a systematic review and meta-analysis. Acta Paediatr. 2015;104(467):96-113.

4. Venancio SI, Escuder MM, Saldiva SR, Giugliani ER. A prática do aleitamento materno nas capitais brasileiras e Distrito Federal: situação atual e avanços. J Pediatr. 2010;86(4):31724.

5. Venancio SI, Saldiva SR, Monteiro CA. Tendência secular da amamentação no Brasil. Rev Saude Publica. 2013;47(6):1205-8.

6. Nguyen PT, Tran HT, Thai T, Foster K, Roberts CL, Marais BJ. Factors associated with breastfeeding intent among mothers of newborn babies in Da Nang, Viet Nam. Int Breastfeed J. 2018;13:2.

7. Odom EC, Li R, Scanlon KS, Perrine CG, Grummer-Strawn L. Reasons for earlier than desired cessation of breastfeeding. Pediatrics. 2013;131(3):e726-32.

8. Moura BL, Alencar GP, Silva ZP, Almeida MF. Internações por complicações obstétricas na gestação e desfechos maternos e perinatais, em uma coorte de gestantes no Sistema Único de Saúde no Município de São Paulo, Brasil. Cad Saude Publica. 2018;34(1):e00188016.

9. Harper A. Reducing morbidity and mortality among pregnant obese. Best Pract Res Clin Obstet Gynaecol. 2015;29(3):427-37.

10. Preusting I, Brumley J, Odibo L, Spatz DL, Louis JM. Obesity as a Predictor of Delayed Lactogenesis II. J Hum Lact. 2017;33(4):684-91.

11. Bever Babendure J, Reifsnider E, Mendias E, Moramarco MW, Davila YR. Reduced breastfeeding rates among obese mothers: a review of contributing factors, clinical considerations and future directions. Int Breastfeed J.. 2015;1:10-21.

12. Oza-Frank R, Moreland JJ, McNamara K, Geraghty SR, Keim SA. Early lactation and infant feeding practices differ by maternal gestational diabetes history. J Hum Lact. 2016;32(4):658-65.

13. Chamberlain C, Wilson A, Amir LH, O'Dea K, Campbell S, Leonard D, et al. Low rates of predominant breastfeeding in hospital after gestational diabetes, particularly among Indigenous women in Australia. Aust N Z J Public Health. 2017;41(2):144-50.

14. EstevesTM, Daumas RP, Oliveira MI,Andrade CA, Leite IC. Fatores associados à amamentação na primeira hora de vida: revisão sistemática. Rev Saúde Pública. 2014;48(4):697-708.

15. Strapasson MR, Ferreira CF, Ramos JG. Feeding practices in the first 6 months after delivery: effects of gestational hypertension. Pregnancy Hypertens. 2018;13:254-9.

16. Instituto Brasileiro de Geografia e Estatística [Internet]. Pesquisa Nacional de Saúde 2013: percepção do estado de saúde, estilos de vida e doenças crônicas: Brasil, grandes regiões e unidades da Federação. Rio de Janeiro: IBGE; 2014 [citado 2018 Nov 9]. Disponível em: https://biblioteca.ibge.gov.br/visualizacao/livros/liv91110.pdf

17. Aiken A, Thomson G. Professionalisation of a breast-feeding peer support service: issues and experiences of peer supporters. Midwifery. 2013;29(12):e145-51.

18. Bueno EB, Coca KP, Abuchaim E, Abrão A, Marcacine K, Draque C. Aleitamento materno exclusivo de recém-nascidos de mães diabéticas em alojamento conjunto. Rev Soc Bras Enferm Ped. 2017;17(2):70-5.

19. Rollins NC, Bhandari N, Hajeebhoy N, Horton S, Lutter CK, Martines JC, et al. Why invest, and what it will take to improve breastfeeding practices? Lancet. 2016;387(10017):491-504.

20. Santana GS, Giugliani ER, Vieira TO, Vieira GO. Fatores associados à manutenção da amamentação por 12 meses ou mais: revisão sistemática. J. Pediatr. 2018;94(2):104-22.

21. Coca KP, Pinto VL, Westphal F, Mania PN, Abrao AC. Bundle of measures to support intrahospital exclusive breastfeeding: evidence of systematic reviews. Rev Paul Pediatr. 2018;36(2):214-20. 
22. Boccolini CS, Carvalho ML, Oliveira MI. Fatores associados ao aleitamento materno exclusivo nos primeiros seis meses de vida no Brasil: revisão sistemática. Rev Saúde Pública. 2015;49:91.

23. Cavalcanti SH, Caminha MF, Figueiroa JN, Serva VM, Cruz RS, Lira PI, et al. Fatores associados à prática do aleitamento materno exclusivo por pelo menos seis meses no estado de Pernambuco. Revista Bras Epidemiol. 2015;18(1):208-19.

24. Wenzel D, Souza SB. Fatores associados ao aleitamento materno nas diferentes regiões do Brasil. Rev Bras Saúde Matern Infant. 2014;14(3):241-9.

25. Meyerink RO, Marquis GS. Breastfeeding initiation and duration among low-income women in Alabama: the importance of personal and familial experiences in making infant-feeding choices. J Hum Lact. 2002;18(1):38-45.
26. Brasil. Ministério da Saúde. Secretaria de Atenção à Saúde Estratégicas. Bases para a discussão da política nacional de promoção, proteção e apoio ao aleitamento materno [Internet]. Brasília: Ministério da Saúde; 2017. [citado 2018 Nov 9]. Disponível em: http:// bvsms.saude.gov.br/bvs/publicacoes/bases_discussao_politica_aleitamento_materno.pdf

27. Almeida JM, Luz SA, Ued FV. Apoio ao aleitamento materno pelos profissionais de saúde: revisão integrativa da literatura. Rev Paul Pediatr. 2015;33(3):356-63.

28. Lawrence RM. Circumstances when breastfeeding is contraindicated. Pediatr Clin North Am. 2013;60(1):295-318.

29. Sachs HC, Frattarelli DA, Galinkin JL, Green TP, Johnson T, Neville K, et al. The transfer of drugs and therapeutics into human breast milk: an update on selected topics. Pediatrics. 2013;132(3):e796-809. 